\title{
The one-dimensional long-range ferromagnetic Ising model with a periodic external field
}

\author{
Azer Kerimov* \\ Bilkent University, Department of Mathematics, 06800 Bilkent, Ankara, Turkey
}

\section{A R T I C L E I N F O}

\section{Article history:}

Received 12 September 2011

Received in revised form 7 November 2011

Available online 18 January 2012

\section{Keywords:}

Ground state

Gibbs state

Extreme Gibbs state

Phase transition

\begin{abstract}
A B S T R A C T
We consider the one-dimensional ferromagnetic Ising model with very long-range interaction under a periodic, biased and weak external field and prove that at sufficiently low temperatures the model has a unique limiting Gibbs state.
\end{abstract}

(c) 2012 Elsevier B.V. All rights reserved.

\section{Introduction}

Over the last few decades numerous rigorous mathematical theories of ferromagnetic behavior have evolved. These theories serve to provide a better understanding of seemingly curious properties of ferromagnetic systems, and their scalar behavior and hysteresis phenomena. The simplest models used to describe such systems are Ising spins with a ferromagnetic pair interaction potential of dipole type. The Ising model has been used to archetype a variety of interacting systems exhibiting cooperative phenomena ranging from ferromagnetism to rather complicated spin glasses. One-dimensional systems with finite range or rapidly decreasing interaction naturally do not exhibit phase transitions at all finite temperature values [1-3]: in the absence of an external field the behavior of spins is dominated by spin waves or magnons. In contrast, in the case of a sufficiently strong pair potential at temperatures below the Curie temperature the phenomenon of spontaneous breaking of global symmetry occurs and two ordered spin states appear in the absence of an additional external field [4,5]. As physically expected, under an identically oriented external field the phenomenon of spontaneous symmetry breaking disappears [6,7]. In the present paper the ferromagnetic model under a less dominant biased and differently oriented external field is explored. It is shown that if the absolute value of the external field is sufficiently weak then, along with the fading out of the local behavior of the field variables, the cooperative activities arising exterminate the spontaneous magnetization.

Let us consider the one-dimensional ferromagnetic Ising model with long-range interaction:

$$
H_{0}(\phi)=-\sum_{x, y \in \mathbf{Z}^{1} ; x>y} U(x-y) \phi(x) \phi(y)
$$

where the spin variables $\phi(x)$ associated with the one-dimensional lattice sites $x$ take the values -1 and +1 and the pair potential $U(x-y)=(x-y)^{-\gamma}, 1<\gamma \leq 2$. The condition $\gamma>1$ provides the existence of the thermodynamical limit and if $\gamma \leq 2$ does not hold, $\sum_{x \in \mathbf{Z}^{1}, x>0} x U(x)<\infty$ and the model (1) has a unique Gibbs state at all temperatures [1-3]. It is well

\footnotetext{
* Tel.: +90 312 2901627; fax: +90 3122664579.

E-mail address: kerimov@fen.bilkent.edu.tr.
} 
known that at sufficiently low temperatures there are extremal Gibbs states $P^{+}$and $P^{-}$corresponding to the ground states $\phi=+1$ and $\phi=-1$ (see Refs. [4,5] for the case $1<\gamma<2$ and [8] for the borderline case $\gamma=2$ ). These sophisticated results are related to the phenomenon of "surface tension" in one dimension. Other profound advances including results on the relation between Fortuin-Kasteleyn percolation and magnetization were obtained for the borderline case $\gamma=2$ $[9,10]$ (for a detailed approach to the random cluster models, see Refs. [11,12]). An alternative approach to the investigation of ferromagnetic systems based on detailed investigation of the geometry of spin configurations is given in Ref. [13] (for $1.5 \leq \gamma \leq 2$ ).

Consider a model (1) under an additional periodic external field:

$$
H(\phi)=H_{0}(\phi)+\sum_{x \in \mathbf{Z}^{1}} h_{x} \phi(x) .
$$

Let $V_{N}$ be an interval with the center at the origin and with the length of $2 N$. We denote the set of all configurations $\phi\left(V_{N}\right)$ by $\Phi(N)$. We denote the concatenation of the configurations $\phi\left(V_{N}\right)$ and $\phi^{i}\left(\mathbf{Z}^{1}-V_{N}\right)$ by $\chi: \chi(x)=\phi(x)$ if $x \in V_{N}$ and $\chi(x)=\phi^{i}(x)$ if $x \in \mathbf{Z}^{\mathbf{1}}-V_{N}$. Define

$$
H_{N}\left(\phi \mid \phi^{i}\right)=\sum_{x, y \in \mathbf{Z}^{1}, x>y,\{x, y\} \cap V_{N} \neq \emptyset} U(x-y)(\chi(x)-\chi(y))+\sum_{x \in \mathbf{Z}^{1}, x \in V_{N}} h_{x} \chi(x) .
$$

The finite-volume Gibbs distribution corresponding to the boundary conditions $\phi^{i}$ is

$$
\mathbf{P}_{\mathbf{N}}^{\mathbf{i}}\left(\phi \mid \phi^{i}\right)=\frac{\exp \left(-\beta H_{N}\left(\phi \mid \phi^{i}\right)\right)}{\Xi\left(N, \phi^{i}\right)}
$$

where $\beta$ is the inverse temperature and the partition function $\Xi\left(N, \phi^{i}\right)=\sum_{\phi \in V_{N}} \exp \left(-\beta H_{N}\left(\phi \mid \phi^{i}\right)\right)$. A probability measure $\mathbf{P}$ on the configuration space $\{-1,1\}^{\mathbf{Z}^{1}}$ is said to be an infinite-volume Gibbs state if for each $N$ and for $\mathbf{P}$ almost all $\phi^{i}$ in $\{-1,1\}^{\mathbf{Z}^{\mathbf{1}}}$ we have

$$
\mathbf{P}\left(\phi\left(V_{N}\right)=\varphi\left(V_{N}\right) \mid \phi\left(\mathbf{Z}^{\mathbf{1}}-V_{N}\right)=\phi^{i}\left(\mathbf{Z}^{\mathbf{1}}-V_{N}\right)\right)=\mathbf{P}_{\mathbf{N}}^{\mathbf{i}}\left(\varphi \mid \phi^{i}\right) .
$$

Below, we investigate the set of all infinite-volume Gibbs states of the model (2). Naturally, in the following known cases the very biased external field exterminates the long-range interaction, the dependence on the boundary conditions disappears at infinity and the model has a unique Gibbs state.

Case 1 . The values of the external field at all lattice points are aligned [6,7]. In this case the infinite-volume Gibbs state is unique at all values of the temperature. This result follows from the ferromagnetic nature of the interaction and uses Fortuin-Ginibre-Kasteleyn or Griffiths-Hurst-Sherman inequalities.

Case 2. The absolute value of the external field is sufficiently big [14]:

Theorem 1. At any fixed value of the inverse temperature $\beta$ there exists a constant $h_{0}$ such that for all realizations of the external field $\left\{h_{x}, x \in \mathbf{Z}^{\mathbf{1}}\right\}$ satisfying $\left|h_{x}\right|>h_{0}, x \in \mathbf{Z}^{\mathbf{1}}$, the model (2) has a unique infinite-volume Gibbs state.

Case 3. The value of the external field is small but the field is "very ordered". Let us consider the periodic external field constituted by alternating (+) and (-) blocks: $h_{x}=h_{x}^{r}$ is a periodic function of period $2 r ; h_{x}^{r}=h_{x+2 r k}$ for all integer values of $k$ and for some fixed positive $\epsilon$,

$$
h_{x}^{r}= \begin{cases}+\epsilon & \text { if } x=1, \ldots, r \\ -\epsilon & x=r+1, \ldots, 2 r .\end{cases}
$$

Theorem 2 ([15]). Let $\epsilon$ be an arbitrary positive fixed number not exceeding some constant $h_{1}$. There exist natural numbers $R_{1}=R_{1}(\epsilon)$ and $R_{2}=R_{2}(\epsilon), R_{1} \leq R_{2}$, such that at all sufficiently small temperatures the model (3) has at least two limiting Gibbs states for all $r \leq R_{1}$ and a unique infinite-volume Gibbs state for all $r>R_{2}$.

Case 4 . The external field is a periodic biased external field. Let $h_{x}=h_{x}^{L}$ be a periodic function of period $3 L$ : for all integer values of $k$ and $n$,

$$
h_{x}^{L}= \begin{cases}\epsilon & \text { if } x=3 k L \text { or } x=(3 k+1) L \\ -\epsilon & \text { if } x=(3 k+2) L \\ 0 & x \neq n L\end{cases}
$$

where $L$ is a positive constant and $0<\epsilon<U(1)$.

Theorem 3. For any values of the positive constants $\epsilon$ and $L$ at sufficiently small values of the temperature, the model (2) with external field (3) has a unique infinite-volume Gibbs state. 
In general, the additional nonsymmetric external field $h_{x}^{L}$ breaks the symmetry between $(+)$ and $(-)$ phases and leads to a unique zero-temperature phase; the statement of Theorem 3 is physically to be expected. But in general the uniqueness of the zero-temperature phase cannot guarantee the uniqueness at nonzero temperatures [16].

In this paper we consider a periodic biased external field. Let $L$ be a positive constant, $h_{x}=h_{x+L}$ for all $x \in \mathbf{Z}^{1}$ and $\sum_{x \in \mathbf{Z}^{1}, 0<x \leq L} h_{x} \neq 0$.

Theorem 4. There exists a constant $h_{0}$ depending on the potential function $U(\cdot)$ and $L$ such that for all realizations of the external field $\left\{h_{x}, x \in \mathbf{Z}^{1}\right\}$ satisfying $\left|h_{x}\right|<h_{0}, h_{x}=h_{x+L}$ and $\sum_{x \in \mathbf{Z}^{1}, 0<x \leq L} h_{x} \neq 0$, for all $x \in \mathbf{Z}^{1}$, the model (2) has a unique infinitevolume Gibbs state at all sufficiently small values of the temperature.

Most likely, the condition $\left|h_{x}\right|<h_{0}$ is essential:

Lemma $1([15])$. Let $h^{\prime}$ be any fixed positive number and the restriction of the external field to $[1, L]$ be a constant configuration: $h_{x}([1, L])=h^{\prime}$. A natural number $L_{0}$ exists such that if $L>L_{0}$, the restriction of any ground state $\phi^{g r}$ of the model to $[1, L]$ is a constant configuration: $\phi^{\text {gr }}([1, L])=h^{\prime}$.

Let the value of $h_{0}$ be fixed. Consider the model (2) with the following periodic external field with period $8 L: h_{x}=h_{x+8 L}$ and

$$
h_{x}^{L}= \begin{cases}-h_{0} & \text { if } 0<x \leq L \text { and } x \neq L / 2 \\ h_{0} & \text { if } x=L / 2 \\ (-1)^{x} h_{0} & \text { if } L<x \leq 4 L \\ h_{0} & \text { if } 4 L<x \leq 5 L \\ (-1)^{x+1} h_{0} & \text { if } 5 L<x \leq 8 L .\end{cases}
$$

It can be readily seen that by Lemma 1, at sufficiently large values of $L$ the model has the following two ground states $\phi^{g r,+}$ and $\phi^{g r,-}$ :

$$
\begin{aligned}
& \phi^{g r,+}= \begin{cases}-h_{0} & \text { if } 0<x \leq L \\
h_{0} & \text { if } L<x \leq 8 L\end{cases} \\
& \phi^{g r,-}= \begin{cases}-h_{0} & \text { if } 0<x \leq 4 L \text { or } 5 L<x \leq 8 L \\
h_{0} & \text { if } 4 L<x \leq 5 L .\end{cases}
\end{aligned}
$$

Most likely, at large temperatures these ground states generate two extreme limiting Gibbs states as in Refs. [4,5]. But the rigorous proof seems to be technically very complicated. Thus, if we consider the model (2) with external field (4) and change the value of $h_{0}$, then the model has unique Gibbs states for sufficiently big and sufficiently small values of $h_{0}$ while it has at least two extreme Gibbs states at intermediate values of $h_{0}$.

\section{Proof of the uniqueness}

We prove the uniqueness of Gibbs states by using a method employing the close relationship between phase transitions and some special kind of combined percolation in models with a unique ground state [17]. The method uses the trick of "coupling" two independent partition functions and is based on the method used in Ref. [18]. Similar "coupling" arguments were used in the disagreement percolation approach to the Gibbs states uniqueness problem $[19,20]$.

Let $\mathbf{P}^{\mathbf{1}}$ and $\mathbf{P}^{\mathbf{2}}$ be two extreme limiting Gibbs states corresponding to the fixed boundary conditions $\phi^{1}$ and $\phi^{2}$. It is well known that $\mathbf{P}^{\mathbf{1}}$ and $\mathbf{P}^{\mathbf{2}}$ are singular with respect to each other or coincide [21,22]. Thus, in order to prove the uniqueness of the limiting Gibbs states we show non-singularity of $\mathbf{P}^{\mathbf{1}}$ and $\mathbf{P}^{\mathbf{2}}$.

If the expression $\left|H_{N}\left(\phi \mid \phi^{i}\right)\right|$ expressing the energy of the configuration $\phi\left(V_{N}\right)$ at fixed boundary conditions $\phi^{i}\left(\mathbf{Z}^{\mathbf{1}}-V_{N}\right)$ is uniformly bounded with respect to $N, \phi$ and $\phi^{i}$, then the non-singularity of $\mathbf{P}^{\mathbf{1}}$ and $\mathbf{P}^{\mathbf{2}}$ follows directly. This idea was used in Ref. [3] for the proof of the absence of a phase transition in one-dimensional models with long-range interaction. But in our case $\left|H_{N}\left(\phi \mid \phi^{i}\right)\right|$ and we employ a more detailed approach.

Let $\phi_{N}^{\min }$ be the configuration with minimal energy at fixed $N$ and boundary conditions $\bar{\phi}$ :

$$
\min _{\phi \in \Phi(N)} H_{N}(\phi \mid \bar{\phi})=H_{N}\left(\phi_{N}^{\min , i} \mid \bar{\phi}\right) \text {. }
$$

$H_{N}\left(\phi \mid \phi^{i}, \phi_{N}^{\min }\right)$ denotes the relative energy of a configuration $\phi$ (with respect to $\phi_{N}^{\min }$ ):

$$
H_{N}\left(\phi \mid \phi^{i}, \phi_{N}^{\min }\right)=H_{N}\left(\phi \mid \phi^{i}\right)-H_{N}\left(\phi_{N}^{\min } \mid \phi^{i}\right) .
$$

Let $\mathbf{P}_{\mathbf{N}}^{\mathbf{i}}$ be Gibbs distributions on $\Phi(N)$ corresponding to the boundary conditions $\phi^{i}, i=1,2$, defined by using relative energies of configurations and $\mathbf{P}_{\mathbf{N}}^{\mathbf{i}} \phi^{\prime}\left(V_{M}\right)$ be the probability of the event that the restriction of the configuration $\phi\left(V_{N}\right)$ to $V_{M}$ coincides with $\phi^{\prime}\left(V_{M}\right)$ for $M<N$. Using the uniqueness of $\phi_{N}^{\text {min }}$ we construct one contour model for both boundary conditions $\phi^{i}, i=1,2$, and after that we come to clusters that are "noninteracting" at distance [23] (a cluster is a collection 
of contours connected by interaction bonds). The cornerstone of the method is the evaluation of the dependence of the expression $\mathbf{P}_{\mathbf{N}}^{\mathbf{1}}\left(\phi\left(V_{M}\right)\right) / \mathbf{P}_{\mathbf{N}}^{\mathbf{2}}\left(\phi\left(V_{M}\right)\right)$ on the boundary conditions $\phi^{1}$ and $\phi^{2}$ in terms of the statistical weights of long clusters connecting the cube $V_{M}$ with the boundary. At low temperatures the application of this theory to one-dimensional models produces a uniqueness criterion [17] which is given below (Theorem 5). A ground state $\phi^{\text {gr }}$ satisfies the Peierls stability condition with a positive constant $\tau$ if for any finite set, $A \subset \mathbf{Z}^{\mathbf{1}} H\left(\phi^{\prime}\right)-H\left(\phi^{g r}\right) \geq \tau|A|$, where $|A|$ denotes the number of sites of $A$ and $\phi^{\prime}$ is a perturbation of $\phi^{\text {gr }}$ on the set $A$.

Condition 1. The model has a unique ground state satisfying the Peierls stability condition.

Condition 2. A constant $\alpha<1$ exists such that for any number $L$ and any interval $I=[a, b]$ with the length $n$ and for any configuration $\phi(I)$,

$$
\sum_{B \subset \mathbf{Z}^{1} ; B \cap l \neq \emptyset, B \cap\left(Z^{1}-[a-L, b+L]\right) \neq \emptyset}|U(B)| \leq \text { const } n^{\alpha} L^{\alpha-1} .
$$

Theorem 5 ([17]). Suppose that a one-dimensional model with a finite spin space and with the translationally invariant Hamiltonian

$$
H(\phi)=\sum_{B \subset \mathbf{Z}^{1}} U(\phi(B))
$$

where $\sum_{B \subset \mathbf{Z}^{1} ; x \in B}|U(B)|<$ const satisfies Conditions 1 and 2. Then a value of the inverse temperature $\beta_{1}$ exists such that if $\beta>\beta_{1}$ then the model has a unique limiting Gibbs state.

Without loss of generality we suppose that $\sum_{x \in \mathbf{Z}^{1}, 0<x \leq L} h_{x}>0$. We can treat the model (2) with an external field with period $L$ as a translationally invariant model: if we partition the lattice into disjoint intervals $[k L+1,(k+1) L]$ and replace the spin space $\{1,-1\}$ by $\{1,-1\}^{[1, L]}$ including $2^{L}$ elements, then the model transforms from being translationally periodic with period $L$ to a translationally invariant model. Thus, for the employing of Theorem 5 , we have to control the validity of Condition 1.

Lemma 2. Suppose that a periodic external field $\left\{h_{x}, x \in \mathbf{Z}^{1}\right\} ; h_{x}= \pm h_{0}$, satisfying $\sum_{x \in \mathbf{Z}^{1}, 0<x \leq L} h_{x}>0$ is fixed. There exists a positive constant $c_{0}$ such that for all values of $h_{0}<c_{0}$ the constant configuration $\phi^{+}=+1$ is the unique ground state of the model (2) and this configuration satisfies the Peierls stability condition.

Proof. We omit the detailed proof of the lemma: it can be readily shown that $c_{0}=\frac{U(1)}{2 L}$ satisfies all conditions.

Condition 2 is readily shown to hold for a pair potential $U(x-y)=(x-y)^{-\gamma}(1<\gamma \leq 2)$ of the model (2). Now the model (2) satisfies Condition 1, and Theorem 4 follows from Theorem 5.

\section{Concluding remarks}

Theorem 4 has a straightforward generalization to periodic external fields taking more than two values.

Theorem 4 states that the cooperative effect of a biased weak periodic external field exterminates the existence of Gibbs states corresponding to two pure ground states. It is noteworthy that when we increase the absolute value of this external field, while maintaining unchanged cooperativeness, local effects of the external field arise and this may lead to the regeneration of two extreme Gibbs states (see example (4)).

\section{Acknowledgments}

The author thanks the referees for helpful comments.

\section{References}

[1] R.L. Dobrushin, Theor. Prob. Appl. 18 (1968) 201.

[2] R.L. Dobrushin, Func. Anal. Appl. 2 (1968) 44

[3] D. Ruelle, Comm. Math. Phys. 9 (1968) 267.

[4] F. Dyson, Comm. Math. Phys. 12 (1969) 91.

[5] F. Dyson, Comm. Math. Phys. 21 (1971) 269.

[6] J.L. Lebowitz, A. Martin-Löf, Comm. Math. Phys. 25 (1972) 276.

[7] C.J. Preston, Comm. Math. Phys. 35 (1974) 253.

[8] J. Frohlich, T. Spencer, Comm. Math. Phys. 84 (1982) 91.

[9] M. Aizenman, C.M. Newman, 107, 611, 1986.

[10] M. Aizenman, J.T. Chayes, L. Chayes, C.M. Newman, J. Stat. Phys. 50 (1988) 1.

[11] H-O. Georgii, O. Häggström, C. Maes, Phase Transitions and Critical Phenomena, 18, Academic Press, 2001, 1.

[12] G. Grimmett, The Random Cluster Model, Springer, 2006.

[13] M. Cassandro, P.A. Ferrari, I. Merola, E. Presutti, J. Math. Phys. 46 (2005) 053305. 
[14] A. Kerimov, IJMPB 17 (2003) 5781.

[15] A. Kerimov, J. Phys. A 40 (2007) 10407.

[16] M. Biskup, L. Chayes, N. Crawford, J. Stat. Phys. 122 (2006) 1139.

[17] A. Kerimov, J. Phys. A 35 (2002) 5365.

[18] A. Kerimov, J. Stat. Phys. 72 (1993) 571.

[19] J. Van den Berg, Comm. Math. Phys. 152 (1993) 161.

[20] J. Van den Berg, C. Maes, Ann. Probab. 22 (1994) 749.

[21] Ya.G. Sinai, Theory of Phase Transitions: Rigorous Results, Pergamon Press, Oxford, 1982.

[22] H-O. Georgii, Gibbs Measures and Phase Transitions, De Gruyter, Berlin, 1988.

[23] J. Bricmont, K. Kuroda, J.L. Lebowitz, Comm. Math. Phys. 101 (1985) 501. 